\title{
Metodologia Fast-Track como ferramenta de qualidade assistencial no enfrentamento da pandemia pela COVID-19
}

\author{
Fast-Track methodology as a quality care tool in the fight against the pandemic by COVID-19 \\ Metodología Fast-Track como herramienta de atención de calidad en la lucha contra la pandemia \\ por COVID-19
}

\author{
Amauri dos Santos Araujo \\ ORCID: https://orcid.org/0000-0001-7435-5670 \\ Universidade Federal de Alagoas, Brasil \\ E-mail: amauriaraujo.sms@gmail.com \\ Isabel Comassetto \\ ORCID: https://orcid.org/0000-0002-2389-9384 \\ Universidade Federal de Alagoas, Brasil \\ E-mail: isabelcomassetto@gmail.com \\ Jovânia Marques de Oliveira e Silva \\ ORCID: https://orcid.org/0000-0001-7452-2651 \\ Universidade Federal de Alagoas, Brasil \\ E-mail: jovaniasilva@gmail.com \\ Elizabeth Moura Soares de Souza \\ ORCID: https://orcid.org/0000-0002-5889-8197 \\ Universidade Federal de Alagoas, Brasil \\ E-mail: elmososo@gmail.com \\ Lenira Maria Wanderley Santos de Almeida \\ ORCID: https://orcid.org/0000-0002-3598-6098 \\ Universidade Federal de Alagoas, Brasil \\ E-mail: leniramsalmeida@gmail.com \\ Thaís Honório Lins Bernardo \\ ORCID: https://orcid.org/0000-0002-8058-8400 \\ Universidade Federal de Alagoas, Brasil \\ E-mail: thais.bernardo@eenf.ufal.br
}

\begin{abstract}
Resumo
O fortalecimento na conjectura para o enfrentamento da pandemia pela COVID-19 no contexto da Atenção Primária à Saúde, vislumbra a necessidade de ferramentas para agilizar a assistencial de forma estratégica, promovendo qualidade na assistência através da organização do fluxo de pacientes de acordo com os recursos disponíveis na abordagem da síndrome gripal. Assim, este estudo objetiva relatar a experiência operacional da metodologia FastTrack como ferramenta que promove qualidade assistencial em serviços de saúde para o enfrentameto da pandemia pela COVID-19. Com esta percepectiva, foi realizado um estudo descritivo, de abordagem qualitativa, do tipo relato de experiência, sobre a operacionalidade da metodologia Fast-Track como ferramenta de qualidade assistencial. Para o adequado funcionamento dos serviços de saúde, a implementação da metodologia garantie agilidade no atendimento, em especial, nos momentos de grandes fluxos assistenciais. Contudo, para atingir a garantia da qualidade e segurança do cuidado, é necessário seguir o padrão operacional em rigor metodológico, para a qualidade do cuidado. Neste sentido, os serviços de saúde passam a receber uma roupagem diferenciada para o atendimento e observação dos pacientes. Assim sendo e, posta em visibilidade pela eficácia e agilidade do funcionamento dos serviços de saúde, a metodologia Fast-Track destaca-se frente ao acolhimento de casos suspeitos no contexto da Atenção Primária à Saúde. Ressalta-se ainda, que o seguimento do cuidado é expresso por sentimentos que permeiam de satisfação pelo acolhimento à classificação final e desfecho dos casos, bem como, estimulando a busca de melhorias da gestão e do cuidado.
\end{abstract}

Palavras-chave: Metodologia; Atenção primária à saúde; Pandemia.

\begin{abstract}
The strengthening of the conjecture for coping with the pandemic by COVID-19 in the context of Primary Health Care highlights the need for tools to strategically streamline care, promoting quality of care by organizing the flow of patients according to resources available in the approach of the flu syndrome. Thus, this study aims to report the operational experience of the Fast-Track methodology as a tool that promotes quality of care in health services to fight the pandemic by COVID-19. With this insight, a descriptive study, with a qualitative approach, of the experience report type, was carried out on the operation of the Fast-Track methodology as a care quality tool. For the proper
\end{abstract}


functioning of health services, the implementation of the methodology ensures agility in care, especially in times of large assistance flows. However, to achieve the assurance of quality and safety of care, it is necessary to follow the operational standard in methodological rigor, for the quality of care. In this sense, health services start to receive a different look for the care and observation of patients. Therefore, and put in visibility by the effectiveness and agility of the functioning of health services, the Fast-Track methodology stands out in relation to the reception of suspected cases in the context of Primary Health Care. it is expressed by feelings that permeate satisfaction with the reception of the final classification and outcome of cases, as well as encouraging the search for improvements in management and care.

Keywords: Methodology; Primary health care; Pandemic.

\section{Resumen}

El fortalecimiento de la conjetura para el afrontamiento de la pandemia por COVID-19 en el contexto de la Atención Primaria de Salud destaca la necesidad de herramientas para agilizar estratégicamente la atención, promoviendo la calidad de la atención a través de la organización del flujo de pacientes según los recursos disponibles en la gestión. del síndrome de la gripe. Así, este estudio tiene como objetivo reportar la experiencia operativa de la metodología Fast-Track como una herramienta que promueve la calidad de la atención en los servicios de salud para combatir la pandemia por COVID-19. Con este insight, se realizó un estudio descriptivo, con enfoque cualitativo, del tipo relato de experiencia, sobre el funcionamiento de la metodología Fast-Track como herramienta de calidad asistencial. Para el buen funcionamiento de los servicios de salud, la implementación de la metodología asegura agilidad en la atención, especialmente en épocas de grandes flujos asistenciales. Sin embargo, para lograr el aseguramiento de la calidad y seguridad de la atención, es necesario seguir el estándar operativo en rigor metodológico, para la calidad de la atención. En este sentido, los servicios de salud comienzan a recibir un aspecto diferenciado para el cuidado y observación de los pacientes. Por tanto, y puesta en visibilidad por la eficacia y agilidad del funcionamiento de los servicios de salud, la metodología Fast-Track se destaca en relación a la recepción de casos sospechosos en el contexto de Atención Primaria de Salud, se expresa en sentimientos que impregnan la satisfacción de la recepción de la clasificación final y el resultado de los casos, así como, estimular la búsqueda de mejoras en la gestión y la atención.

Palabras clave: Metodología; Primeros auxilios; Pandemia.

\section{Introdução}

O presente estudo tem como objeto o registro do Fast-Track da abordagem sindrômica de Síndrome Gripal nos atendimentos aos cidadãos com suspeita de COVID-19. Compreendendo à Atenção Primária à Saúde (APS) como papel fulcral para a implementação das ações protetivas e preventivas, a ampliação metodológica e conceitual de métodos, busca reforçar o fortalecimento na conjectura para resolução, prevenção e promoção à saúde, no enfrentamento da pandemia pela COVID-19 (Araujo; Comassetto, 2021; Dunlop et al., 2020; Souza \& Souza, 2020; Sarti et al., 2020).

Vale destacar, que a implementação de protocolos para o processo de trabalho permite p acesso e o desenvolvimento do cuidado no contexto da pandemia. Morley et al. (2020), Nurciaroni et al. (2020), Sarti et al. (2020) e Araujo \& Comassetto (2021), ainda destacam que esses são alguns dos desafios para o fortalecimento da APS em face à alta transmissibilidade e letalidade em um contexto social cosmopolítico.

Deste modo, após a descoberta do novo coronavírus em 31 de dezembro de 2019, a Organização Mundial da Saúde (OMS) declara a disseminação do vírus Sarv-CoV-2, que provocou o surto identificado pandêmico veiculado como pandemia da COVID-19. Partindo desse cenário, uma força tarefa foi estruturada para a implementação de estratégias e ações para a contenção e redução da infecção pelo vírus que assola o mundo (Souza \& Souza, 2020; OMS, 2020a ; Araujo; Comassetto, 2021; Silva; Araujo, 2021).

Diante o contexto pandêmico, foi declarada pela Organização Mundial de Saúde (OMS) em 30 de janeiro de 2020, como uma Emergência de Saúde Pública de Importância Internacional - ESPII, e, partindo de atos regulatórios, o Ministério da Saúde (MS), por meio da Portaria Ministerial no 188, de 03/02/2020, considerou a mesma como sendo uma Emergência em Saúde Pública de importância Nacional - ESPIN (Brasil, 2020; OMS, 2020a; WHO, 2020).

Neste ínterim, tais estratégias para o enfrentamento do Sarv-CoV-2, tem apresentado respostas efetivas no cenário assistencial. Como a metodologia de registro do Fast-Track da abordagem sindrômica de Síndrome Gripal contribui na 
organização dos atendimentos aos cidadãos com suspeita de COVID-19? Face ao questionamento, emerge o seguinte objetivo: relatar a experiência operacional da metodologia de registro do Fast-Track contribui como ferramenta na organização de qualidade assistencial em serviços de saúde para a abordagem sindrômica de Síndrome Gripal aos cidadãos com suspeita de COVID-19.

\section{Metodologia}

Trata-se de um estudo descritivo, de abordagem qualitativa, do tipo relato de experiência, sobre a operacionalidade da metodologia Fast-Track como ferramenta de qualidade assistencial. Com a necessidade de roupagem dos serviços de saúde no contexto da APS no enfrentamento da pandemia pela COVID-19, municípios e estados intensificam forças para o desenvolvimento de ações estratégicas que versatilizem habilidades e competências com efetividades para a mitigação dos casos.

Nesse sentido, a APS tem como papel fulcral, reorganizar a assistência nos moldes de Unidades de Triagem para Síndromes Gripais (UTSG) como forma de garantir os direitos dos pacientes com casos suspeitos e/ou confirmados da infecção pelo vírus Sars-CoV-2. Logo, estas UTSG apresentam destaques ao seguir com uma metodologia que orienta o cuidado frente ao episódio de emergência sanitária pela pandemia viral, sendo capazes de promover o controle do novo coronavírus e evitar complicações graves do SARS-CoV-2 (Dunlop et al., 2020; Souza \& Souza, 2020; Sarti et al., 2020; Araujo \& Comassetto, 2021).

Reitera-se que este relato de experiência operacional se torna essencial para compreensão acerca da importância do uso de metodologias que integram as estratégias e ações para a organização de serviços de saúde como ferramenta de qualidade assistencial e inovadora do cuidado em tempos de pandemia.

\section{Resultados e Discussão}

A pandemia pela COVID-19 trouxe perspectivas de mudança para o contexto assistencial. Tais mudanças propiciam preocupações para as autoridades sanitárias em relação as demandas existentes - atendimentos, sobrecarga dos profissionais de saúde e pacientes com casos suspeitos ou confirmados - na comunidade a ser assistida. O Instituto de Pesquisa Econômica Aplicada (Ipea, 2020), ainda destaca que esses aspectos são de importância para a segurança e qualidade do cuidado realizado pela equipe que está atrelada a construção, reestruturação e modificação da importância social dos profissionais, bem como, da identidade no processo.

Destarte, há ainda a necessidade da implementação das precauções padrão, que a constitui como a principal medida de prevenção contra a transmissão entre pacientes e profissionais de saúde, devendo ser adotada em todos os serviços e abranger o cuidado de todos os pacientes no serviço - na chegada, triagem, espera e durante toda assistência; independentemente dos fatores de risco ou doença de base, garantindo que as políticas e práticas internas possam minimizar a exposição à COVID-19 (Brasil, 2020a; Brasil, 2020b).

Morley et al. (2020), Nurciaroni et al. (2020), Sarti et al. (2020), destacam que esses são alguns dos desafios para o fortalecimento da APS em face à alta transmissibilidade e letalidade em um contexto social cosmopolítico. Logo, vale destacar, que a implementação de metodologias para o processo de trabalho facilita o desenvolvimento do cuidado no contexto da pandemia.

Para o adequado funcionamento dos serviços de saúde na APS, a implementação da metodologia Fast-Track foi um marco para a garantia de agilidade no atendimento, em especial, nos momentos de grandes fluxos assistenciais. Contudo, para atingir a garantia da qualidade e segurança do cuidado, é necessário seguir o padrão em rigor metodológico (Sarti et al., 2020; Souza \& Souza, 2020). 


\section{Metodologia Fast-Track como Ferramenta de Qualidade do Cuidado no Contexto da Atenção Primária à Saúde}

Meios para o enfrentamento da pandemia pela COVID-19 em articulação com ações de atenção à saúde no contexto da APS, foram traçadas. Diante o exposto, cabe ressaltar que a necessidade de estabelecer a readaptação dos serviços de saúde para o acolhimento e triagem dos casos, estratégias como a implementação da metodologia Fast-Track, foi inserida no cenário para uma melhor assistencial aos casos suspeitos e confirmados da COVID-19, objetivando garantir a qualidade do cuidado a ser prestado a comunidade, e face a elevada transmissibilidade do vírus.

A metodologia Fast-Track, com ferramenta de qualidade do cuidado, apresenta operacionalização e funcionalidade em passos simples. Em primeira instância, ainda na recepção da unidade de saúde, o paciente é abordado pelo Agente de Acolhimento para identificação das necessidades. Diante à necessidade de agilizar o fluxo, neste momento é oportunizada à higienização das mãos com álcool à 70\% em gel, dispensação de máscaras para os pacientes que se apresentem ao serviço sem o devido uso e realizada à aferição de temperatura com termômetro digital - infravermelho. Ressalta-se que este agente deve ser profissional de nível médio técnico em enfermagem devidamente treinado para a abordagem ao público-alvo (Jansen et al., 2020; Souza \& Souza, 2020; Soares et al., 2020), conforme representado pela Figura 1.

Destaca-se que para a implementação deste tipo de metodologia, se faz necessária a implementação de educação permanente para segurança e qualidade da assistência a ser prestada durante a operacionalização. Logo, englobam aspectos que identificam as necessidades do paciente na chegada ao serviço e passam a ser avaliados a partir de um bundle (Ficha cadastral). Após o acolhimento, o paciente é encaminhado para o devido preenchimento da bunble e, posteriormente, triagem/avaliação de sinais vitais e avaliação/conduta pelo Enfermeiro a partir da sintomatologia clínica apresentada, caracterizando o segundo passo. Em seguida, após esta avaliação, o paciente é encaminhado para o consultório médico - enquanto em um dos consultórios o médico atende o paciente que foi avaliado pela equipe de enfermagem, em outro consultório, se faz o primeiro atendimento do próximo paciente, caracterizando assim, o terceiro passo (Araujo; Comassetto, 2021; Jansen et al., 2020).

Feito o atendimento de acordo com a necessidade e condição clínica do paciente, segue-se com à realização da classificação final conforme definições operacionais para diagnóstico e orientações na plataforma do sistema e-SUS Notifica: confirmado por critério clínico, laboratorial em indivíduo assintomático, laboratorial em não vacinado e vacinados contra COVID-19, clínico-imagem, confirmado clínico-epidemiológico, caso de síndrome gripal não específica ou descartado para COVID-19; assim, o médico direciona para sala de medicação e/ou dispensação de medicação na farmácia, realização de exames laboratoriais e de imagem que julgar necessário frente a condição clínica ou é encaminhado para alta com orientações para o isolamento domiciliar ou não, e logo, é redirecionado para recepção, e encerra-se o quarto passo de operacionalização do cuidado (Barbosa et al., 2020; Jansen et al., 2020; Soares et al., 2020). 
Figura 1 - Fluxo Fast-Track de operacionalização em Unidade de Triagem para Síndromes Gripais.

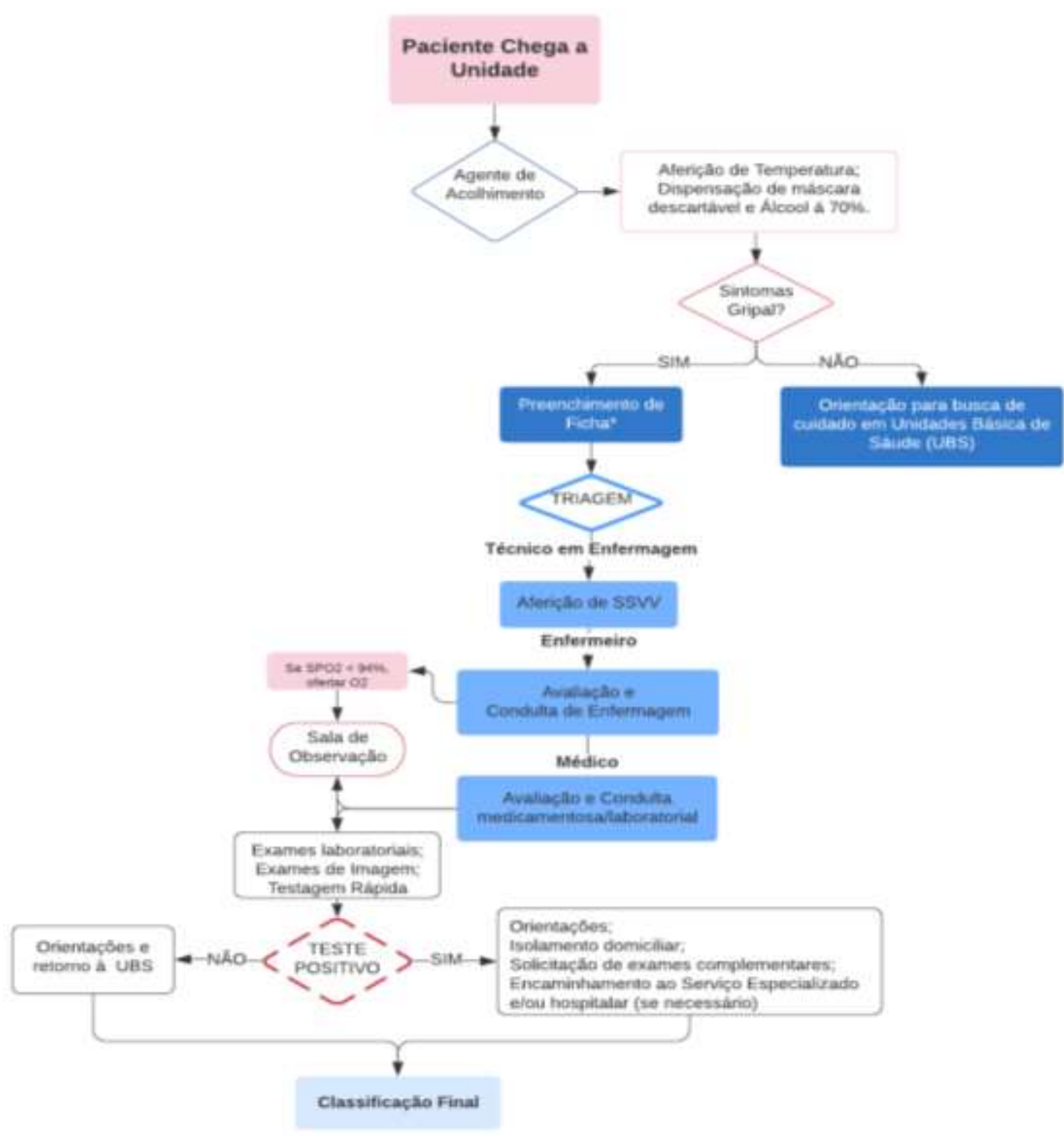

Fonte: Autores.

De acordo com os critérios estabelecidos e seguidos no passo a passo durante o acolhimento, à implementação de testes rápidos antígeno anticorpo, a avaliação é realizada de acordo com a necessidade da conduta e critérios estabelecidos no manejo clínico e oitavo dia de sintomatologia. Reitera-se que para o desenvolvimento das atividades frente à metodologia, fazse necessário um quadro de enfermeiros e técnicos em enfermagem, técnicos em laboratório, auxiliares administrativos e de farmácia, farmacêuticos, médicos, além de direção e apoio de direção (Jansen et al., 2020; Moura; Mundim-Pombo; Oliveira, 2020; Soares et al., 2020).

Outrossim, para a efetividade das ações detalhadas é imprescindível à oferta de oxigênio medicamentoso para os casos que precisam de aporte suplementar do gás medicinal. Diante da necessidade de cuidados de abrangência no contexto da APS frente às ações para as respostas emergenciais no enfrentamento do SARVS-CoV-2, este tipo de metodologia como ferramenta de qualidade assistencial tem sido referência no manejo às respostas emergenciais para o enfrentamento da nova pandemia (Menezes et al., 2020; Nunciaroni et al., 2020; Souza \& Souza, 2020). 
O cuidado de enfermagem diante do Novo Coronavírus no contexto da APS restabelece a força presente no sistema de saúde. Em se tratando de ações, a resposta em Redes de Atenção à Saúde (RAS) como principal porta de entrada do sistema é capaz de ser altamente resolutiva para diferentes situações de saúde, logo, faz-se necessário a garantia e efetividades de práticas seguras para o território (Jansen et al., 2020; Menezes et al., 2020; Moura; Mundim-Pombo; Oliveira, 2020; Nunciaroni et al., 2020; Souza \& Souza, 2020).

Outrossim, é fundamental que a APS seja reconhecida e receba financiamento adequado para (re)criar processos de cuidado tanto às pessoas acometidas pela COVID-19 como para o acompanhamento longitudinal de saúde nos territórios continuamente. Dessa forma, será possível (re)construir a saúde pública brasileira vilipidiada por meio do fortalecimento do SUS.

\section{Conclusão}

A interface do cenário assistencial requer o olhar longitudinal do cuidado para o enfrentamento e controle da pandemia pela COVID-19. Destarte, vale salientar, que a implementação de estratégias de registro do Fast-Track contribui como uma ferramenta organizacional de qualidade assistencial para a abordagem sindrômica de Síndrome Gripal - um disparador de possibilidades destinadas a comunidade. No entanto, os aspectos que envolvem a assistência ao paciente nos dos serviços de saúde necessitam seguir orientações e protocolos estabelecidos com o propósito de prevenção de doenças e manutenção da saúde, até o restabelecimento da mesma.

Assim sendo e, posta em visibilidade pela eficácia e agilidade do funcionamento dos serviços de saúde, a metodologia Fast-Track destaca-se frente a uma garantia de triagem mais rápida e eficiente, possibilitando uma adequação do espaço ás necessidades da demanda e a manutenção das medidas de prevenção de casos suspeitos de COVID-19 no contexto da Atenção Primária à Saúde. Ressalta-se ainda, que o seguimento do cuidado é expresso por sentimentos que permeiam de satisfação pelo acolhimento à classificação final e desfecho dos casos, bem como, estimulando a busca de melhorias da gestão e do cuidado.

\section{Referências}

Araujo, A.S.; Comassetto, I. The nursing protagonism in the organization of health services during the COVID-19 pandemic. Research, Society and Development, 2021, v. 10, n. 1, e48110112014.

Brasil (2020a). Painel Coronavírus. Ministério da Saúde. Brasília, DF.

Brasil (2020b). Portaria nº 188, de 3 de Fevereiro de 2020. Ministério da Saúde. Brasília, DF.

Dunlop, C. et al. (2020). The coronavirus outbreak: the central role of primary care in emergency preparedness and response. BJGP Open.

Jansen, R. et al. Contribuições da rede de pesquisa em processo de enfermagem para assistência na pandemia de COVID-19. Rev Brasileira de Enfermagem, 2020, v.73 (Suppl 2), p:e20200798.

Menezes, H.F. et al. Terminologia especializada de enfermagem para à prática clínica à COVID-19. Texto \& Contexto Nursing, 2020, v. 29: e20200171.

Morley, G. et al. (2020). Covid-19: Ethical Challenges for Nurses. Hastings Center Report. 50(Issue 3), 35-9.

Moura, R.F.; Mundim-Pombo, A.P.M.; Oliveira, J.F.M. Novo Coronavírus (2019-nCoV): Análise da magnitude nos dois primeiros meses da epidemia. Revista Nursing, 2020, v.23, n.266, p.:4330-4335.

Nunciaroni, À.T. et al Novo Coronavírus: (re)pensando o processo de cuidado na Atenção Primária à Saúde e a Enfermagem. Rev. Bras. Enferm., Brasília , v. 73, supl. 2, e20200256, 2020 .

Sarti, T.D. et al. (2020). Qual o papel da Atenção Primária à Saúde diante da pandemia provocada pela COVID-19? Epidemiol. Serv. Saude, Brasília, v. 29, n. 2, e2020166.

Silva, I. N. da; Araujo, A. dos S. Psychological and physical impacts on professional nurses in coping with the COVID-19 pandemic. Research, Society and Development, [S. 1.], v. 10, n. 8, p. e41410815695, 2021. DOI: 10.33448/rsd-v10i8.15695.

Soares, S.S.S. et al. De cuidador a paciente: na pandemia da Covid-19, quem defende e cuida da enfermagem brasileira?. Esc. Anna Nery, Rio de Janeiro, 2020, v.24, n.spe, e20200161. 
Research, Society and Development, v. 10, n. 12, e556101220755, 2021

(CC BY 4.0) | ISSN 2525-3409 | DOI: http://dx.doi.org/10.33448/rsd-v10i12.20755

Souza \& Souza, L.P. Atenção primária à saúde no Brasil: resultados, avanços e desafios. Campo Grande: Editora Inovar, 2020. 573p.

Soeiro, R.E. et al. (2020). Atenção Primária à Saúde e a pandemia de COVID-19: reflexão para a prática. InterAm J Med Heath, Campinas, v. 3, e202003010. 